\title{
Visualization of Scenarios for the Transition of Oscillations from Harmonic to Chaotic for a Micropolar Kirchhoff-Love Cylindrical Meshed Panel
}

\author{
E.Yu. Krylova' ${ }^{1}$ I.V. Papkova ${ }^{2}$, O. A. Saltykova²,V.A. Krysko ${ }^{2}$ \\ Kat.krylova@bk.rulikravzova@mail.ru|olga_a_saltykova@mail.ru|tak@sun.ru \\ ${ }^{1}$ Saratov State University, Saratov, Russia; \\ ${ }^{2}$ Yuri Gagarin State Technical University of Saratov, Saratov, Russia;
}

\begin{abstract}
On the basis of the kinematic hypotheses of the Kirchhoff-Love built a mathematical model of micropolar cylindrical meshed panels vibrations under the action of a normal distributed load. In order to take into account the size-dependent behavior, the panel material is considered as a Cosser's pseudocontinuum with constrained particle rotation. The mesh structure is taken into account by the phenomenological continuum model of G. I. Pshenichnov. For a cylindrical panel consisting of two systems of mutually perpendicular edges, a scenario of transition of oscillations from harmonic to chaotic is constructed. It is shown that in the study of the behavior of cylindrical micropolar meshed panels it is necessary to study the nature of the oscillations of longitudinal waves.
\end{abstract}

Keywords:visualization of scenarios for the transition of oscillations into chaos, a mesh structure, a cylindrical panel, micropolar theory, the Kirchhoff-Love model.

\section{Introduction}

To solve the problems of static and dynamic mesh plates, panels and shells, mainly two computational models are used. It is a phenomenological continuum model and a discrete model. In the continuum model, a mesh object consisting of a regular system of frequently located edges of one material is replaced by an equivalent solid object having some averaged stiffness depending on the arrangement of the edges and their stiffness $[1,3]$. In the discrete model, the edges are represented by beam, shell, or three-dimensional finite elements [2,5,7,11]. Eachoftheseapproacheshasitsadvantages [4].

Progress in micro-and nano-technologies leads to the interest of scientists not only to the behavior of full-size mechanical systems in the form of plates and shells $[13,14,16]$, but also the need to create mathematical models that take into account the scale effects at the micro and nano level $[10,12,19]$. In most works on this subject linear models are used for numerical analysis $[15,17,18,21,22]$. However, there are experimental data confirming the need to take into account the nonlinearity in modeling the behavior of the objects under consideration [20].

Despite the large number of works devoted to the sizedependent behavior of mechanical objects in the form of plates, panels and shells, studies of the behavior of mesh plates and shells based on theories that take into account the effects of scale is very small $[6,8,9]$.

\section{Problemstatement}

A mathematical model of oscillations of a micro-polar flexible rectangular cylindrical panel under the action of a transverse distributed pressure occupying a region in space $R^{3}$ area $\Omega=\left\{0 \leq x \leq c ; 0 \leq y \leq b ;-\frac{h}{2} \leq z \leq \frac{h}{2}\right\}$ is constructed.

The panel consists $n$ of sets of densely arranged edges of the same material, which allows the use of a phenomenological continuum model. Taking into account the Kirchhoff-love hypotheses, the strain tensor components are written as:

$e_{x x}=\frac{\partial u}{\partial x}+\frac{1}{2}\left(\frac{\partial w}{\partial x}\right)^{2}-z \frac{\partial^{2} w}{\partial x^{2}} ; e_{y y}=\frac{\partial v}{\partial y}+\frac{1}{2}\left(\frac{\partial w}{\partial x}\right)^{2}-k_{y} w-z \frac{\partial^{2} w}{\partial y^{2}} ;$

$e_{x y}=\frac{1}{2}\left(\frac{\partial u}{\partial y}+\frac{\partial v}{\partial x}\right)+\frac{\partial w}{\partial x} \frac{\partial w}{\partial y}-z \frac{\partial^{2} w}{\partial x \partial y}$.

Where $u, v, w$ - axial displacements of the middle surface of the plate in the directions $x, y, z$ respectively, $k_{y}$ - geometric curvature parameter.
To account for the size-dependent behavior, a non-classical continuum model based on the Cosser medium is considered, where, along with the usual stress field, torque stresses are also taken into account. This assumes that the displacement and rotation fields are not independent. In this case, the components of the symmetric bending-torsion tensor are written as follows:

$$
\begin{aligned}
& \chi_{x x}=\frac{\partial^{2} w}{\partial x \partial y} ; \chi_{y y}=-\frac{\partial^{2} w}{\partial y \partial x} ; \chi_{x y}=\frac{1}{2}\left(\frac{\partial^{2} w}{\partial y^{2}}-\frac{\partial^{2} w}{\partial x^{2}}\right) ; \\
& \chi_{x z}=\frac{1}{4}\left(\frac{\partial^{2} v}{\partial x^{2}}-\frac{\partial^{2} u}{\partial x \partial y}\right) ; \chi_{y z}=\frac{1}{4}\left(\frac{\partial^{2} v}{\partial y \partial x}-\frac{\partial^{2} u}{\partial y^{2}}\right) .
\end{aligned}
$$

We take the defining relations for the panel material in the form:

$$
\left(m_{x x}, m_{x y}, m_{z x}\right)=\frac{E l^{2}}{1+v}\left(\chi_{x x}, \chi_{x y}, \chi_{z x}\right),
$$$$
\sigma_{x x}=\frac{E}{1-v^{2}}\left[e_{x x}+v e_{y y}\right], x \square \quad y, \sigma_{x y}=\frac{E}{(1+v)} e_{x y} \text {, где } \sigma_{i j} \quad-\text { the }
$$

components of the stress tensor, $m_{i j}$ components of the moment tensor of higher order, E - Young's modulus, $v$ - Poisson's ratio.

The equations of motion of a smooth plate element equivalent to a mesh one, boundary and initial conditions are obtained from the Hamilton - Ostrogradsky energy principle:

$$
\begin{aligned}
& \frac{\partial N_{x x}}{\partial x}+\frac{\partial T}{\partial y}+\frac{1}{2} \frac{\partial^{2} Y_{y z}}{\partial y^{2}}+\frac{1}{2} \frac{\partial^{2} Y_{x z}}{\partial x \partial y}=\rho h \frac{\partial^{2} u}{\partial t^{2}}, \\
& \frac{\partial N_{y y}}{\partial y}+\frac{\partial T}{\partial x}-\frac{1}{2} \frac{\partial^{2} Y_{x z}}{\partial x^{2}}-\frac{1}{2} \frac{\partial^{2} Y_{y z}}{\partial x \partial y}=\rho h \frac{\partial^{2} v}{\partial t^{2}}, \\
& \frac{\partial N_{x x}}{\partial x} \frac{\partial w}{\partial x}+N_{x x} \frac{\partial^{2} w}{\partial x^{2}}+\frac{\partial N_{y y}}{\partial y} \frac{\partial w}{\partial y}+N_{y y} \frac{\partial^{2} w}{\partial y^{2}}+2 \frac{\partial^{2} H}{\partial x \partial y}+2 \frac{\partial T}{\partial x} \frac{\partial w}{\partial y}+ \\
& +2 \frac{\partial T}{\partial y} \frac{\partial w}{\partial x}+4 T \frac{\partial^{2} w}{\partial x \partial y} \frac{\partial^{2} M_{x x}}{\partial x^{2}}+\frac{\partial^{2} M_{y y}}{\partial y^{2}}+2 \frac{\partial^{2} H}{\partial x \partial y}+k_{y} N_{y y}- \\
& -\frac{\partial^{2} Y_{x x}}{\partial x \partial y}+\frac{\partial^{2} Y_{y y}}{\partial y \partial x}+\frac{\partial^{2} Y_{x y}}{\partial x^{2}}-\frac{\partial^{2} Y_{x y}}{\partial y^{2}}+2 q=\rho h \frac{\partial^{2} w}{\partial t^{2}}+\varepsilon \rho h \frac{\partial w}{\partial t} .
\end{aligned}
$$




$$
\begin{aligned}
& \left\{N_{x x} \frac{\partial w}{\partial x}+\frac{\partial M_{x}}{\partial x}+2 T \frac{\partial w}{\partial y}+\frac{\partial Y_{x y}}{\partial x}+2 \frac{\partial H}{\partial y}-\frac{\partial Y_{x x}}{\partial y}+\frac{\partial Y_{y y}}{\partial y}-\frac{\partial Y_{x y}}{\partial x}\right\}_{n_{x}}+ \\
& +\left\{N_{y y} \frac{\partial w}{\partial y}+\frac{\partial M_{y}}{\partial y}+2 T \frac{\partial w}{\partial x}+2 \frac{\partial H}{\partial x}-\frac{\partial Y_{x x}}{\partial x}+\frac{\partial Y_{y y}}{\partial x}-\frac{\partial Y_{x y}}{\partial y}\right\}_{n_{y}}=0 \\
& \left\{M_{x x}-Y_{x y}\right\}_{n_{x}}+\left\{-2 H+Y_{x x}-Y_{y y}\right\}_{n_{y}}=0 \\
& \left\{-2 H+Y_{x x}-Y_{y y}\right\}_{n_{x}}+\left\{-M_{y y}+Y_{x y}\right\}_{n_{y}}=0 \\
& \left\{N_{x x}-\frac{1}{2} \frac{\partial Y_{x z}}{\partial y}\right\}_{n_{x}}+\left\{T-\frac{1}{2} \frac{\partial Y_{y z}}{\partial y}-\frac{1}{2} \frac{\partial Y_{x z}}{\partial x}\right\}_{n_{y}}=0 ; \\
& \left\{N_{x y}-\frac{1}{2} \frac{\partial Y_{x z}}{\partial x}-\frac{1}{2} \frac{\partial Y_{y z}}{\partial y}\right\}_{n_{x}}+\left\{N_{y y}-\frac{1}{2} \frac{\partial Y_{y z}}{\partial x}\right\}_{n_{y}}=0 ;\left\{\frac{1}{2} Y_{x z}\right\}_{n_{y}}=0 \\
& \left\{Y_{x z}\right\}_{n_{x}}+\left\{Y_{y z}\right\}_{n_{y}}=0 ;\left\{\frac{1}{2} Y_{x z}\right\}_{n_{x}}+\left\{\frac{1}{2} Y_{y z}\right\}_{n_{y}}=0 ;\left\{\frac{1}{2} Y_{y z}\right\}_{n_{x}}=0 .
\end{aligned}
$$

Here the expression for the classical force and torque:

$$
\begin{aligned}
& \left(N_{x x}, N_{y y}, T\right)=\int_{-\frac{h}{2}}^{\frac{h}{2}}\left(\sigma_{x x}, \sigma_{y y}, \sigma_{x y}\right) d z, \\
& \left(M_{x x}, M_{y y}, H\right)=\int_{-\frac{h}{2}}^{\frac{h}{2}}\left(\sigma_{x x}, \sigma_{y y}, \sigma_{x y}\right) z d z,
\end{aligned}
$$

as well as expressions for the forces caused by instantaneous stresses:

$$
Y_{x x}=\int_{-\frac{h}{2}}^{\frac{h}{2}} m_{x x} d z, \quad Y_{x y}=\int_{-\frac{h}{2}}^{\frac{h}{2}} m_{x y} d z, \quad Y_{x z}=\int_{-\frac{h}{2}}^{\frac{h}{2}} m_{x z} d z, \quad x \square \quad y .
$$

The stresses arising in the equivalent smooth panel associated with the stresses in the edges that make up the angles $\varphi_{j}$ with the abscissa axis will have the form: $\sigma_{x y}=\sum_{j=1}^{n} \frac{\sigma_{x}^{j} \delta_{j} \operatorname{Cos} \varphi_{j} \operatorname{Sin} \varphi_{j}}{a_{j}}, \sigma_{x x}=\sum_{j=1}^{n} \frac{\sigma_{x}^{j} \delta_{j} \operatorname{Cos}^{2} \varphi_{j}}{a_{j}}$, $\sigma_{y y}=\sum_{j=1}^{n} \frac{\sigma_{x}^{j} \delta_{j} \operatorname{Sin}^{2} \varphi_{j}}{a_{j}}, \quad m_{x y}=\sum_{j=1}^{n} \frac{m_{x}^{j} \delta_{j} \operatorname{Cos} \varphi_{j} \operatorname{Sin} \varphi_{j}}{a_{j}}$, $m_{x x}=\sum_{j=1}^{n} \frac{m_{x}^{j} \delta_{j} \operatorname{Cos}^{2} \varphi_{j}}{a_{j}}, \quad m_{y y}=\sum_{j=1}^{n} \frac{m_{x}^{j} \delta_{j} \operatorname{Sin}^{2} \varphi_{j}}{a_{j}}$, $m_{x z}=\sum_{j=1}^{n} \frac{m_{z x}^{j} \delta_{j} \operatorname{Cos} \varphi_{j}}{a_{j}}, \quad m_{y z}=\sum_{j=1}^{n} \frac{m_{z x}^{j} \delta_{j} \operatorname{Sin} \varphi_{j}}{a_{j}}$, where $a_{j}$ distance between edges of $\mathrm{j}$-th family, $\delta_{j}$ - the thickness of the ribs, voltage index $\mathrm{j}$ are rods. The physical relations for the mesh plate are determined based on the Lagrange multiplier method: $\sigma_{x}^{j}=\sigma_{x x} \operatorname{Cos}^{2} \varphi_{j}+\sigma_{y y} \operatorname{Sin}^{2} \varphi_{j}+\sigma_{x y} \operatorname{Cos} \varphi_{j} \operatorname{Sin} \varphi_{j} ;$

$\tau^{j}=\sigma_{x z} \operatorname{Cos} \varphi_{j}+\sigma_{y z} \operatorname{Sin} \varphi_{j} ;$

$m_{x}^{j}=m_{x x} \operatorname{Cos}^{2} \varphi_{j}+m_{y y} \operatorname{Sin}^{2} \varphi_{j}+m_{x y} \operatorname{Cos} \varphi_{j} \operatorname{Sin} \varphi_{j} ;$

$m_{z}^{j}=m_{x z} \operatorname{Cos} \varphi_{j}+m_{y z} \operatorname{Sin} \varphi_{j}$.

Subject to designation: $A_{s k}=\sum_{j=1}^{n} \frac{\delta_{j} \operatorname{Cos}^{s} \varphi_{j} \operatorname{Sin}^{k} \varphi_{j}}{a_{j}} ; s, k=\overline{0,4}$ expressions for classical forces and moments, as well as the forces caused by the moment stresses of the cylindrical mesh panel will take the form: (the upper index shows the account of the mesh structure):

$$
\begin{aligned}
& N_{x x}^{s}=A_{40} N_{x x}+A_{22} N_{y y}+A_{31} T ; \\
& N_{y y}^{s}=A_{22} N_{x x}+A_{04} N_{y y}+A_{13} T ; \\
& T^{s}=A_{31} N_{x x}+A_{13} N_{y y}+A_{22} T ; \\
& M_{x x}^{s}=A_{40} M_{x x}+A_{22} M_{y y}+A_{31} H ; \\
& M_{y y}=A_{22} M_{x x}+A_{04} M_{y y}+A_{13} H ; \\
& H^{s}=A_{31} M_{x x}+A_{13} M_{y y}+A_{22} H ; \\
& Y_{x x}^{s}=A_{40} Y_{x x}+A_{22} Y_{y y}+A_{31} Y_{x y} ; \\
& Y_{y y}^{s}=A_{22} Y_{x x}+A_{04} Y_{y y}+A_{13} Y_{x y} ; \\
& Y_{x y}^{s}=A_{31} Y_{x x}+A_{13} Y_{y y}+A_{22} Y_{x y} ; \\
& Y_{y z}^{s}=A_{11} Y_{x z}+A_{02} Y_{y z}+A_{01} Y_{z z} ; \\
& Y_{x z}^{s}=A_{20} Y_{x z}+A_{11} Y_{y z}+A_{10} Y_{z z} .
\end{aligned}
$$

Substituting expressions (2) into equations (1), we obtain a resolving system of equations of motion for a smooth micropolar cylindrical Kirchhoff-Love panel equivalent to the original mesh panel.

In this model, the rigidity of the rods to bend in a plane tangent to the middle surface of the panel is not taken into account, so the orders of systems of differential equations describing the behavior of grid and solid panels coincide. At the same time, the formulations of the boundary conditions of the corresponding boundary value problems coincide.

The scenario of transition of oscillations from harmonic to chaotic cylindrical panel with two families of edges is investigated $\varphi_{1}=45^{\circ}, \varphi_{2}=135^{\circ}, \delta_{1}=\delta_{2}=\delta, a_{1}=a_{2}=a(\mathrm{Fig}$ .1). Taking into account dimensionless parameters: $u=\frac{h^{2}}{c} \bar{u}$, $v=\frac{h^{2}}{b} \bar{v}, \quad k_{y}=\frac{h}{b^{2}} \overline{k_{y}}, \quad q=\frac{E h^{4}}{c^{2} b^{2}} \bar{q} ; t=b \sqrt{\frac{\rho}{E}}-\bar{t}, \quad \varepsilon=\frac{1}{b} \sqrt{\frac{E}{\rho}} \bar{\varepsilon}$, $x=c \bar{x}, y=b \bar{y}, w=h \bar{w}, \delta=h \bar{\delta}, a=h \bar{a}, l=h \bar{l}$, where $\varepsilon$ dissipation factor, $\rho$ - the density of the panel material, $q=q_{0} \operatorname{Sin}\left(\omega_{p} t\right)$ - external normal load, $q_{0}$ and $\omega_{p}$ - its intensity and frequency, $t$ - time. The equations of motion of the element of the considered micropolar mesh cylindrical panel will take the form (the line above the dimensionless variables is omitted):

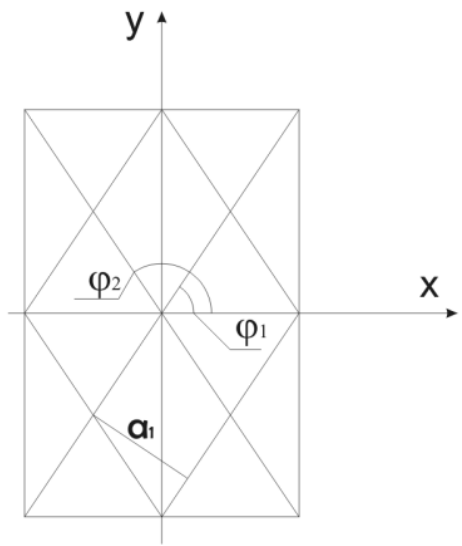

Fig.1.Panel mesh geometry. 


$$
\begin{aligned}
& +l^{2}(v-1)\left(-\frac{h^{2}}{b^{2}} \frac{\partial^{4} u}{\partial y^{4}}+\frac{h^{2}}{b^{2}} \frac{\partial^{4} v}{\partial x \partial y^{3}}-\frac{h^{2}}{c^{2}} \frac{\partial^{4} u}{\partial x^{2} \partial y^{2}}+\frac{h^{2}}{c^{2}} \frac{\partial^{4} v}{\partial x^{3} \partial y}\right)+ \\
& +2(v-1) \frac{\partial^{2} u}{\partial y^{2}}-2(3+v) \frac{\partial^{2} v}{\partial x \partial y}-4(v+1) \frac{b^{2}}{c^{2}} \frac{\partial^{2} u}{\partial x^{2}}- \\
& -4 k_{y}(v+1) \frac{b}{h} \frac{\partial w}{\partial x}+4(v-1) \frac{\partial^{2} w}{\partial y^{2}} \frac{\partial w}{\partial x}-8 \frac{\partial w}{\partial y} \frac{\partial^{2} w}{\partial x \partial y}- \\
& -4(1+v) \frac{b^{2}}{c^{2}} \frac{\partial w}{\partial x} \frac{\partial^{2} w}{\partial x^{2}}=\frac{8 a\left(v^{2}-1\right)}{\delta} \frac{h^{2}}{c^{2}} \frac{\partial^{2} u}{\partial t^{2}} \\
& l^{2}(v-1)\left(\frac{h^{2}}{b^{2}} \frac{\partial^{4} u}{\partial x \partial y^{3}}-\frac{h^{2}}{b^{2}} \frac{\partial^{4} v}{\partial x^{2} \partial y^{2}}+\frac{h^{2}}{c^{2}} \frac{\partial^{4} u}{\partial x^{3} \partial y}-\frac{h^{2}}{c^{2}} \frac{\partial^{4} v}{\partial x^{4}}\right)- \\
& -4 k_{y}(1+v) \frac{c^{2}}{b h} \frac{\partial w}{\partial y}-4(1+v) \frac{c^{2}}{b^{2}} \frac{\partial^{2} v}{\partial y^{2}}-2(3+v) \frac{\partial^{2} u}{\partial x \partial y}- \\
& -2(1-v) \frac{\partial^{2} v}{\partial x^{2}}-4(1+v) \frac{c^{2}}{b^{2}} \frac{\partial w}{\partial y} \frac{\partial^{2} w}{\partial y^{2}}-8 \frac{\partial w}{\partial x} \frac{\partial^{2} w}{\partial y \partial x}+ \\
& +4(v-1) \frac{\partial w}{\partial y} \frac{\partial^{2} w}{\partial x^{2}}=\frac{8 a\left(v^{2}-1\right)}{\delta} \frac{h^{2}}{b^{2}} \frac{\partial^{2} v}{\partial t^{2}} \\
& \left([1+v]+6 l^{2}[1-v]\right) \frac{c^{2}}{b h} \frac{\partial^{4} w}{\partial y^{4}}+4\left(1+l^{2}[v-1]\right) \frac{c^{2}}{b^{2}} \frac{\partial^{4} w}{\partial x^{2} \partial y^{2}}+ \\
& +\left([1+v]+6 l^{2}[1-v]\right) \frac{b^{2}}{c^{2}} \frac{\partial^{4} w}{\partial x^{4}}+12 k_{y}^{2}(1+v) \frac{c^{2}}{b^{2}} w+ \\
& +12 k_{y}(1+v) \frac{c^{2}}{b h} \frac{\partial v}{\partial y}+12 k_{y}(1+v) \frac{b^{2}}{h^{2}} \frac{\partial u}{\partial x}-6 k_{y}(1+v) \frac{c^{2}}{b h}\left(\frac{\partial w}{\partial y}\right)^{2}- \\
& -12(1+v) \frac{c^{2}}{b^{2}} \frac{\partial w}{\partial y} \frac{\partial^{2} v}{\partial y^{2}}-12 k_{y}(1+v) \frac{c^{2}}{b h} w \frac{\partial^{2} w}{\partial y^{2}}-12(1+v) \frac{c^{2}}{b^{2}} \frac{\partial v}{\partial y} \frac{\partial^{2} w}{\partial y^{2}}- \\
& -18(1+v)\left(\frac{\partial w}{\partial y}\right)^{2} \frac{\partial^{2} w}{\partial y^{2}}-12(v+1) \frac{\partial^{2} w}{\partial y^{2}} \frac{\partial u}{\partial x}+12(v-1) \frac{\partial^{2} u}{\partial y^{2}} \frac{\partial w}{\partial x}- \\
& -6 k_{y}(v+1) \frac{b}{h}\left(\frac{\partial w}{\partial x}\right)^{2}+6(-5+3 v) \frac{\partial^{2} w}{\partial y^{2}}\left(\frac{\partial w}{\partial x}\right)^{2}-24 \frac{\partial w}{\partial y} \frac{\partial^{2} u}{\partial x \partial y}- \\
& -24 \frac{\partial w}{\partial x} \frac{\partial^{2} v}{\partial x \partial y}+24(v-1) \frac{\partial u}{\partial y} \frac{\partial^{2} w}{\partial x \partial y}+24(v-1) \frac{\partial v}{\partial x} \frac{\partial^{2} w}{\partial x \partial y}+ \\
& +24(3 v-5) \frac{\partial w}{\partial y} \frac{\partial w}{\partial x} \frac{\partial^{2} w}{\partial x \partial y}-12(1+v) \frac{b^{2}}{c^{2}} \frac{\partial w}{\partial x} \frac{\partial^{2} u}{\partial x^{2}}+ \\
& +12(v-1) \frac{b}{c} \frac{\partial w}{\partial y} \frac{\partial^{2} v}{\partial x^{2}}-12 k_{y}(v+1) \frac{b}{h} w \frac{\partial^{2} w}{\partial x^{2}}-12(v+1) \frac{c^{2}}{b^{2}} \frac{\partial v}{\partial y} \frac{\partial^{2} w}{\partial x^{2}}+ \\
& +6(3 v-5)\left(\frac{\partial w}{\partial y}\right)^{2} \frac{\partial^{2} w}{\partial x^{2}}-18(1+v) \frac{b^{2}}{c^{2}}\left(\frac{\partial w}{\partial x}\right)^{2} \frac{\partial^{2} w}{\partial x^{2}}- \\
& -12(1+v) \frac{\partial u}{\partial x} \frac{\partial^{2} w}{\partial x^{2}}=\frac{24 a\left(v^{2}-1\right)}{\delta}\left(\frac{\partial^{2} w}{\partial t^{2}}+\varepsilon \frac{\partial w}{\partial t}-2 q\right)
\end{aligned}
$$

Boundary conditions - rigid sealing at all ends of the panel: $u=v=w=0, \frac{\partial u}{\partial x}=0, \frac{\partial u}{\partial y}=0, \frac{\partial v}{\partial x}=0, \frac{\partial v}{\partial y}=0,$.

$\frac{\partial w}{\partial x}=0, \frac{\partial w}{\partial y}=0 \quad$ npu $\quad x= \pm 1, y= \pm 1$.

Initial conditions - zero.

The nonlinear partial differential problem in spatial coordinates is reduced to an ordinary differential problem by the finite difference method with the second-order approximation of accuracy. To do this, the derivatives of spatial variables are replaced by finite central differences. The Cauchy time problem is solved by the Runge-Kutta method of the fourth order of accuracy.

\section{Scenarios of transition of oscillations of a cylindrical panel to chaos}

To visualize the scenarios of transition of oscillations of a micropolar mesh cylindrical panel from harmonic to chaotic for deflection and displacement, the following characteristics were constructed and analyzed: signal, Fourier spectrum, wavelet 2D and 3D spectra based on the mother wavelet Morle, phase and modal portraits, signs of largestLyapunov exponents.

The following is a scenario of transition of oscillations of a grid cylindrical micropolar panel from harmonic to chaotic (Table 1-3).

The parameters of the experiment: $l=0.5, c=b=1$, $h=0.2, \varepsilon=1, \delta=a=0.2, \quad v=0.3, \omega_{p}=5, \quad t \in[0 ; 512]$, $q_{0} \in[0 ; 200]$.From the data collected in the tables it can be seen that in addition to the characteristics of the deflection, the nature of the oscillations of the longitudinal waves should be studied, which will allow a more accurate picture of the nature of the oscillations of the system. At load amplitude $q_{0}=0.1$, the Fourier power spectrum for the deflection shows harmonic oscillations, but the Lyapunov exponent for the deflection is positive. This discrepancy is explained by the fact that the signal of the displacement function $\mathrm{u}$ has a chaotic component at low frequencies. Harmonics at the same frequencies are present in the deflection signal, but the Fourier spectrum does not display them. These frequencies demonstrate the wavelet spectrum, so it is necessary to consider the Fourier spectrum and the wavelet spectrum together. As the load increased, a harmonic appeared in the signal at an independent frequency $\omega_{1}$. When the amplitude of the load $q_{0}=190$ phase portrait of the deflection shows chaotic oscillations and the power spectrum of the Fourier transform of the oscillations at two frequencies. Thus, to determine the type of deflection oscillations, it is also necessary to consider the function of moving by $0 \mathrm{x}$ or $0 \mathrm{y}$.

Table1. The characteristics of the deflection function $w$ and the displacement function $\mathrm{u}$

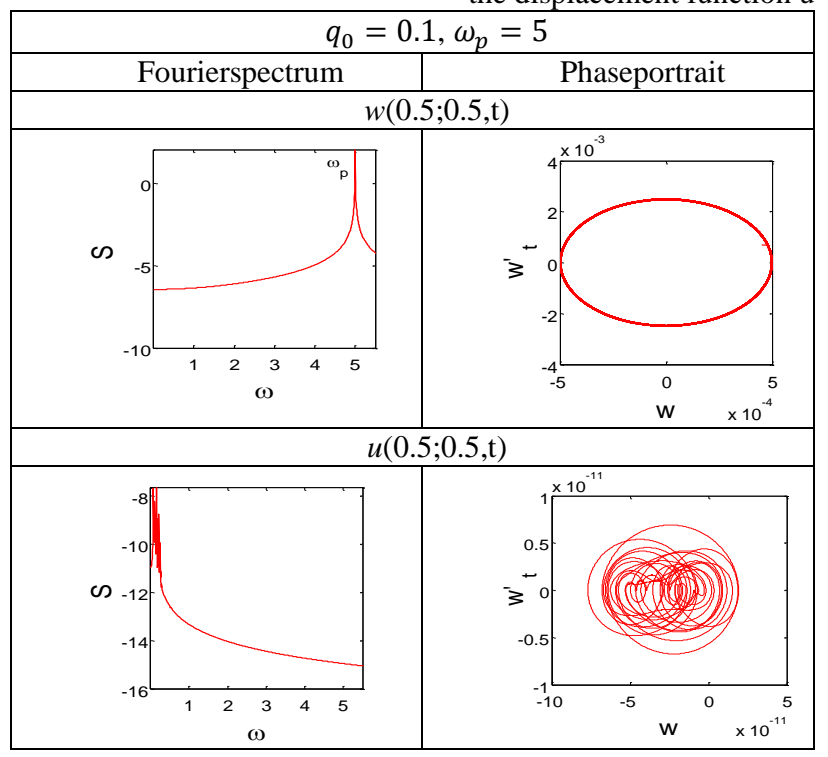

Table2. The characteristics of the deflection function $w$ and the displacement function $\mathrm{u}$ $q_{0}=130, \omega_{p}=5$

Fourierspectrum 


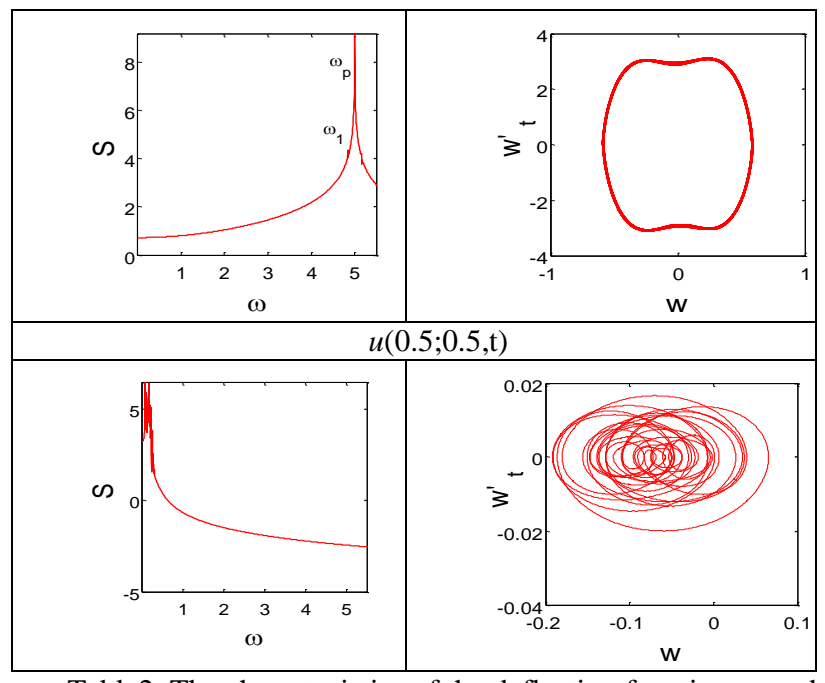

Table2. The characteristics of the deflection function $w$ and the displacement function $\mathrm{u}$

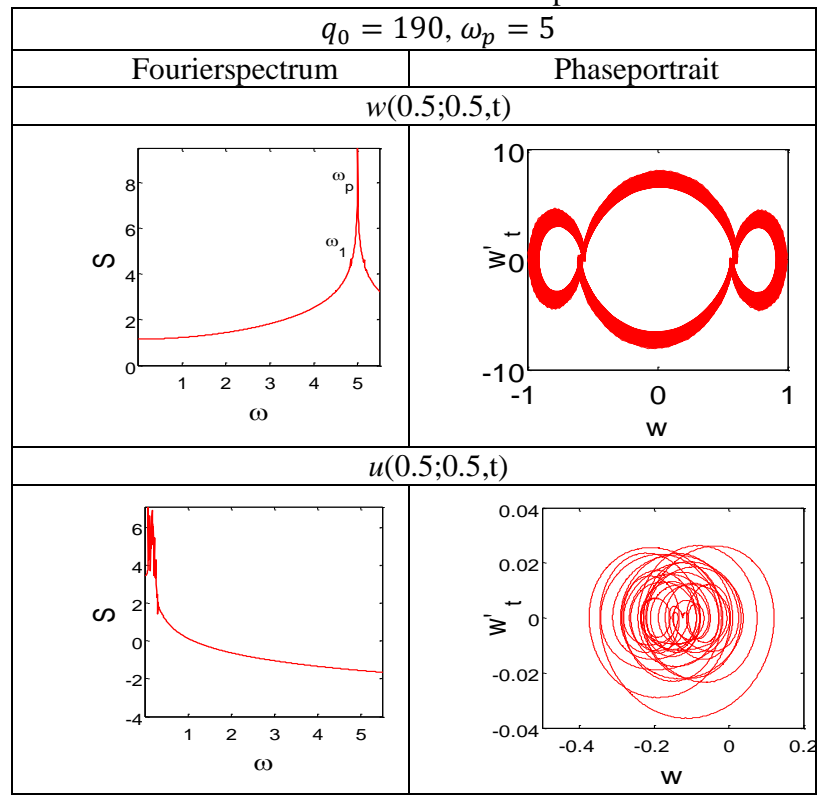

\section{Conclution}

A mathematical model of nonlinear oscillations of a cylindrical panel of a grid structure is constructed. For a deep analysis of the behavior of a micropolar mesh cylindrical panel, it is necessary to visualize the characteristics of not only the deflection function, but also the displacement function, as well as to consider the entire apparatus of nonlinear dynamics in the aggregate.

\section{Acknowledgements}

The study was supported by grants: RFBR №18-01-00351 a and №18-41-700001 r_a.

\section{References}

[1] Azarov A.V. Continuum model of composite mesh shells formed by a system of spiral edges // Composites and nanostructures. 2015. Vol. 7. № 3 (27). P. 151-161. (in Russian) [2] Azikov N. With. Pavlov E. A. stability Study of a mesh composite plate //Aviation industry. 2016. № 3. P. 46-50. (in Russian)

[3] Belikov G. I. General case of bending of a rectangular mesh plate taking into account the tensile forces acting in the middle surface // Bulletin of the Volgograd state University of architecture and civil engineering. Series: Construction and architecture. 2014. № 37 (56). P. 121-128. (in Russian)

[4] Burnysheva T. V., Kaledin V. O. Comparison of discrete and continuous approach to the calculation of the stress state of mesh shell structures under static loading //Journal Scientific and technical of the Volga region. 2011. № 4. P. 113-116. (in Russian)

[5] Burnysheva T. V., Steinbrecher, O. A., Ulyanov A. D. Aspects of specifying boundary conditions in the simulation mesh anisogamy designs // Bulletin of the South Ural state University. Series: Mathematical modeling and programming. 2018. V. 11. № 1. P. 137-144. (in Russian)

[6] Eremeev V. A On a nonlinear model of the mesh shell // Izvestiya of the Russian Academy of Sciences. Solid mechanics. 2018. № 4. P. 127-133 (in Russian)

[7] Zinin A.V., Azik N. With. The model of destruction process of composite structures anisakidae problems //Mechanical engineering and reliability of machines.2018. № 5. C. 49-56. (in Russian)

[8] E. Yu. Krylova, V. I. Papkova, O. A. Saltykova, O. A. Sinichkina, Krys'ko V. A. Mathematical model of the fluctuations of the size-dependent cylindrical shells mesh structure given the hypotheses of the Kirchhoff-Love //non-linear world. 2018. Vol. 16. № 4. P. 17-28(in Russian)

[9] Krylov E. Y., Papkova I. V., Saltykova, O. A., Yakovleva T. V., Krysko V. A.-jr Analysis of the natural frequencies of the micropolar, mesh for cylindrical panel of the Kirchhoff-love In the collection: Problems of mechanical engineering proceedings of the III International scientific-technical conference. In 2 parts. Scientific editor P. D. Balakin. 2019. P. 278-282.(in Russian) [10] NikabadzeM.U. Some variants of the equations of the micropolar theory of shells Applied mathematics and mathematical physics.2015. Vol. 1. № 1. P. 101-118. (in Russian)

[11] Trushin S.I., Zhuravleva T.A., Sysoeva E.V. Dynamic loss of stability of nonlinear deformable grid plates made of composite material with different lattice configurations // Scientific review. 2016. No. 4. P. 44-51.

[12] Awrejcewicz J., Krysko V.A., Sopenko A.A., Zhigalov M.V., Kirichenko A.V., Krysko A.V. Mathematical modelling of physically/geometrically non-linear micro-shells with account of coupling of temperature and deformation fields // Chaos, Solitons \& Fractals. 2017. V. 104. P. 635-654.

[13] Awrejcewicz J., Mrozowski J., Krysko A.V., Papkova I.V., Zakharov V.M., Erofeev N.P., Krylova E.Y., Krysko V.A. Chaotic dynamics of flexible beams driven by external white noise //Mechanical Systems and Signal Processing. 2016. T. 79. C. 225-253.

[14] Krylova E Y, Papkova I V, Erofeev N P, Zakharov V M, Krysko V A Complex fluctuations of flexible plates under longitudinal loads with account for white noise // Journal of Applied Mechanics and Technical Physics. 2016. V. 57. № 4. P. 714-719.

[15] Krylova E Yu, Papkova I V, Sinichkina A O, Yakovleva T B, Krysko-yang V A Mathematical model of flexible dimensiondependent mesh plates // IOP Conf. Series: Journal of Physics: Conf. Series 1210 (2019) 012073 doi:10.1088/17426596/1210/1/012073

[16] Krysko V A, Papkova I V, Awrejcewicz J, Krylova E Y, Krysko A V Non-symmetric forms of non-linear vibrations of flexible cylindrical panels and plates under longitudinal load and additive white noise // Journal of Sound and Vibration. 2018. V. 423. P. 212-229.

[17] Safarpour H., Mohammadi K. and Ghadiri M. Temperaturedependent vibration analysis of a FG viscoelastic cylindrical microshell under various thermal distribution via modified length scale parameter: a numerical solution // Journal of the Mechanical Behavior of Materials 2017, Volume 26, Issue 1-2, Pages 9-24 
[18] Sahmani S., Ansari R., Gholami, R., Darvizeh A. Dynamic stability analysis of functionally graded higher-order shear deformable microshells based on the modified couple stress elasticity theory //Composites Part B Engineering 51 (2013) 4453

[19] Sargsyan S.H., Zhamakochyan K.A. Applied theory of micropolar elastic thin plates with constrained rotation and the finite element method// Materials Physics and Mechanics. 2018. T. 35. № 1. C. 145-154

[20] Scheible D. V., Erbe A., Blick R. H. Evidence of a nanomechanical resonator being driven into chaotic responsevia the Ruelle-Takens route // Appl. Phys. Lett. 81 (2002), 18841886.

[21] Varygina M. Numerical modeling of micropolar cylindrical shells on supercomputers with GPUs // AIP Conference Proceedings 1895, 080005 (2017)

[22] Xinping Zhou Lin Wang Vibration and stability of microscale cylindrical shells conveying fluid based on modified couple stress theory Micro \& Nano Letters 2012 Volume: 7 , Issue: 7 , p $679-684$ 